\title{
Drugs and therapeutics for Global Pharmacology
}

\author{
Takayuki Manabe*
}

Faculty of Nursing, Laboratory for Neuroanatomy and Neuropharmacology, Chyukyogakuin University, Japan

Pharmacology has traditionally studied all life forms from the genome to the individual level, and widely contributes to the fields of medicine and pharmacy as a basic integrated science. This discipline involves basic and clinical interrelationships established by exploring biological phenomena, determining disease conditions, establishing the basis for drug treatment and action, the creation of new drugs, and advanced medication such as tissue regeneration, including research on induced pluripotent stem cells. Pharmacology can thus be rephrased as "the science that establishes the foundation of drug treatment". Pharmacology involves a comprehensive study of the interaction between chemical substances, in vivo and in vitro, and living organisms through their organs, tissues, and cells, and at a molecular level, using various research methods. Furthermore, pharmacological research, such as drug discovery, is conducted from the perspective of disease treatment. Since the 1980s, pharmacological research has been accelerated by the spread of multidisciplinary and cross-sectional studies, including molecular biology and biochemistry. More recently, drug discovery research has been conducted using bioinformatics techniques to analyze genomic information and protein databases. Studies are also conducted at the level of entire organisms, and rather than focusing on one cell or one organ, contemporary pharmacological systems approaches aim to comprehensively understand drug responses by examining cell-cell networks or organ-organ networks.

In recent years, personalized medicine based on genome drug discovery, proteomics approaches, and RNA drug discovery has also progressed. For example, personalized medicine is attracting attention for the development of treatments for mental illnesses involving multiple factors and exhibiting a complex phenotype. There is a trend towards basic research to produce pharmacological and psychogenic clinical data. However, connecting large-scale data to personalized medicine and the discovery of new pathways using the latest bioinformatics technology can assist in the future development of all pharmacological fields.

An important goal in the pharmacological sciences is to produce new medicines by synthesizing results using various research methods. This approach has developed recently under the umbrella term 'translational research', which has been established in the biomedical field, mainly in the areas of cancer and psychiatric diseases. This concept means "bridging research", connecting the results of basic research directly to clinical treatments. This theme has been embraced by many related international journals and has grown into an active research field, and this journal will be no exception.

Therefore, it is possible to say that the adage, 'cannot see the forest for the trees', is not applicable to current pharmacological studies. In this context, in the first issue of this journal the chosen theme is "Global Pharmacology". This inaugural issue aims to broadly show case global pharmacological research that boldly challenges the unknown. In this first issue, we will deal with the latest research concerning traditional Chinese medicine, functional foods and natural substances, as well as new medicines. We hope that researchers involved in basic and clinical research will become readers. Therefore, the areas covered by the journal are extensive. The research methods and approaches used and covered in the included studies are diverse, and include translational research, bioinformatics, and personalized medicine, from fundamental fields such as biology, cellular molecular biology, immunology, and disease state molecular neurobiology. We hope that the first issue provides a general overview, in addition to a deep exploration of specialized areas, and that researchers with various backgrounds will find plenty to interest them.
Copyright: (C2016 Manabe T. This is an open-access article distributed under the terms of the Creative Commons Attribution License, which permits unrestricted use, distribution, and reproduction in any medium, provided the original author and source are credited.
Correspondence to: Takayuki Manabe, Professor, Faculty of Nursing, Laboratory for Neuroanatomy and Neuropharmacology, Chyukyogakuin University, Japan, E-mail: t-manabe@chukyogakuin-u.ac.jp

Received: November 17, 2016; Accepted: November 28, 2016; Published: December 02, 2016 